\title{
Hydrogels for efficient light delivery in optogenetic applications
}

S. Johannsmeier, M. L. Torres, T. Ripken, D. Heinemann, A. Heisterkamp

S. Johannsmeier, M. L. Torres, T. Ripken, D. Heinemann, A. Heisterkamp, "Hydrogels for efficient light delivery in optogenetic applications ," Proc. SPIE 10482, Optogenetics and Optical Manipulation 2018, 104820Q (14 February 2018); doi: $10.1117 / 12.2289470$

SPIE. Event: SPIE BiOS, 2018, San Francisco, California, United States 


\title{
Hydrogels for efficient light delivery in optogenetic applications
}

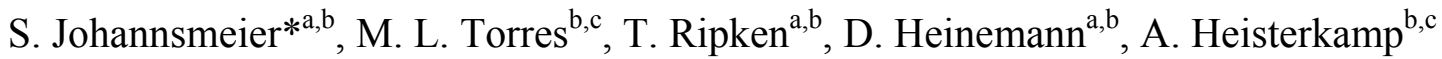 \\ ${ }^{a}$ Industrial and Biomedical Optics Department, Laser Zentrum Hannover e.V, Hollerithallee 8, \\ 30419 Hannover; ${ }^{\mathrm{b}}$ Lower Saxony Centre for Biomedical Engineering, Implant Research and \\ Development (NIFE), Stadtfelddamm 34, 30625 Hannover; ${ }^{c}$ Institute of quantum optics, Gottfried \\ Wilhelm Leibniz Universität Hannover, Welfengarten 1, 30167 Hannover
}

\begin{abstract}
Light-based therapies have been established for various indications, such as skin conditions, cancer or neonatal jaundice. Advances in the field of optogenetics open up new horizons for light-tissue interactions with an organism-wide impact. Excitable tissues, such as nerve and muscle tissues, can be controlled by light after the introduction of light-sensitive ion channels. Since these organs are generally not easily accessible to illumination in vivo, there is an increasing need for effective biocompatible waveguides for light delivery. These devices not only have to guide and distribute the light as desired with minimal losses, they should also mimic the mechanical properties of the surrounding tissue to ensure compatibility. In this project, we are tuning the properties of hydrogels from poly(ethylene glycol) derivatives to achieve compatibility with muscle tissue as well as optimal light guiding and distribution for optogenetic applications at the heart. The excitation light is coupled into the hydrogel with a biocompatible fiber. Properties of the hydrogel are mainly tuned by monomer length and concentration. Total reflection can be achieved by embedding a fiber-like hydrogel with a high refractive index into a second, low refractive index gel. Different geometries and scattering microparticles are used for light distribution in a flat gel patch. Targeted cell attachment can be achieved by introducing a protein layer to the otherwise bioinert gel. After optimization, the hydrogel may be used to deliver light for the excitation of genetically altered cardiomyocytes for controlled contraction.
\end{abstract}

Keywords: optogenetics, hydrogels, light delivery, waveguiding, biohybrid implant, cardiac pacing, defibrillation

\section{INTRODUCTION}

\subsection{Optogenetics}

Since the identification of light-driven ion channels in bacteria and algae, a new toolbox has been developed in biology. By introducing channels such as Channelrhodopsin-2 (ChR-2, an unspecific cation channel) or halorhodopsin (NpHR, permeable for chloride ions) to nerve or muscle cells, it is possible to control their membrane potential via light stimuli, thereby evoking a neuronal response or a contraction. The unprecedented spatio-temporal resolution of this stimulation method has already proven itself a valuable tool in basic research ${ }^{1-3}$. In vivo studies are already testing the versatility and robustness of optogenetics in living organisms ${ }^{4,5}$, paving the way for novel optical therapies that are more gentle and precise than their current counterparts which rely on electrical stimulation.

*s.johannsmeier@1zh.de; www.lzh.de

Optogenetics and Optical Manipulation 2018, edited by Samarendra K. Mohanty, Nitish V. Thakor, E. Duco Jansen, Proc. of SPIE Vol. 10482, 104820Q · C 2018 SPIE · CCC code: 1605-7422/18/\$18 · doi: 10.1117/12.2289470 


\subsection{Cardiac arrhythmia and cardioversion}

In the healthy heart, the excitation stimulus is conducted along specialized paths and quickly distributed to the muscle tissue. This coordination of excitation allows for synchronized contraction of all parts of the heart, and therefore efficient blood ejection. Subsequently, the whole heart is in a refractory state and cannot be excited for a period of approx. $300 \mathrm{~ms}$. Specialized pacemaker cells in the heart's sinus node determine the rhythm of contraction. Failure of these pacemaker cells or disturbances in the conduction paths can cause re-entry cycles - excitation is conducted in twirls, reentering some areas while others are still refractory. Consequently, the heart muscle does not contract as one but in uncoordinated patches. This results in a dramatically reduced ejection volume, a sudden drop of blood pressure, unconsciousness and, if proper blood flow cannot be restored quickly, death. The acute nature of this phenomenon makes it necessary for people at risk to wear an implantable cardioverter defibrillator (ICD), which detects arrhythmias in the patient's heart and triggers an electrical stimulus. This overrides the heart's own excitation cycles and resets the whole muscle to a refractory state, afterwards returning control to the sinus node. In the USA alone, about 800,000 patients live with an $\mathrm{ICD}^{6}$. Risk factors are congenital cardiac arrhythmias but also disturbances in conduction, which can occur after reperfusion following a heart attack. The process of defibrillation itself however poses considerable stress on the muscle tissue and the patient. The event is very painful, leaving some of those affected multiple times with clinical depression or post-traumatic stress disorders ${ }^{7}$. Moreover, the strong electrical shock can itself cause secondary injuries, further impairing correct conduction of excitation. Optical stimulation in combination with introduction of optogenetic channels to the tissue would greatly increase spatial resolution and have a largely reduced impact on the stimulated muscle cells.

\subsection{Hydrogels as implantable waveguides}

Therapies based on optogenetic methods can only be realized with the help of efficient light delivery into living tissues. The employed devices need to guide the stimulating light to the target tissue; a process that is complicated by strong scatter, instability of the immediate surroundings and the need for biocompatibility and possibly long term engraftment. Implantable devices such as $\mu$ LED arrays or fibers already meet some of these requirements. While arrays from $\mu$ LEDs are very flexible, engraftment of these devices into the target tissue is problematic. Biocompatible fibers are useful for spot illumination, but cannot be employed by themselves to target larger tissue areas. Light guiding hydrogels offer great versatility in their optical and mechanical properties ${ }^{8-10}$, can be engrafted into the target tissue ${ }^{11}$ and are already in use for tissue engineering purposes ${ }^{12}$. Hydrogel waveguides are already being tested in live tissues for applications such as sensing or phototherapies ${ }^{9,13}$. In optogenetics, they could prove to be a valuable tool in distributing light to genetically manipulated tissues and enable simultaneous stimulation of a large number of cells.

\subsection{Development of an implantable optical defibrillator}

Combining the need for an alternative therapy for cardiac arrhythmia, the advances in optogenetics and the versatility of hydrogel waveguides, we are developing a novel device for optical cardioversion and defibrillation in a collaborative project. A biocompatible hydrogel shall distribute the light to transgenic engineered heart muscle tissue expressing ChR2 , acting as the starting point for the defibrillating stimulus. The feasibility of this approach has been tested and demonstrated before by ourselves (unpublished) as well as our partners ${ }^{14,15}$. Now, we are aimed at advancing these methods to achieve a robust and reliable long term implant for painless cardioversion and defibrillation. In this paper, strategies for light distribution by hydrogels as well the control of the hydrogel-tissue-interface are described.

\section{METHODS}

\subsection{Fabrication of hydrogels}

Hydrogels were produced from poly(ethylene glycol) diacrylate (PEGDA, MW 700, Sigma Aldrich) or poly(ethylene glycol) dimethacrylate monomers (PEGDMA, MW 8000 or 20,000, Polysciences). The monomers were dissolved in water at the indicated concentration (all given as wt \%). Microparticles were added where indicated (polystyrene particles from Kisker Biotech; $\mathrm{TiO}_{2}$ particles, rutile, from Sigma Aldrich). Then, ammonium persulfate (10\% solution in $\mathrm{H}_{2} \mathrm{O}$ ) and TEMED (both Millipore Sigma) were added at 1:100 or 1:1000, respectively. The mixtures were poured into the desired forms and radicalic polymerization occurred under a nitrogen atmosphere for 45-60 minutes. Gels were then stored in purified water until further use. 
<smiles>C=CC(=O)OCCOC(=O)C=C</smiles><smiles>C=C(C)C(=O)OCC(C)OC(=O)C(=C)C</smiles>

Figure 1: Chemical structure of poly(ethylene glycol) diacrylate (left) and dimethacrylate (right). After radical formation, the acrylate headgroups link the molecules to a network in a chain reaction.

\subsection{Hydrogel measurements}

Power losses were measured with a cut-back method: Inside a silicone mold, a hydrogel of $10 \mathrm{~cm}$ length with a diameter of $800 \mu \mathrm{m}$ was produced. A green laser $(\lambda=532 \mathrm{~nm})$ was coupled into this hydrogel fiber and the output power at the end of the hydrogel was measured. The gel was the shortened by $1 \mathrm{~cm}$ and power was measured again; this procedure was repeated until the end of the gel was reached. From input and output power, the damping coefficient was calculated.

Transmissivity was determined with a photometer. Three hydrogels per monomere were produced inside micro cuvettes and absorption and transmission were measured in the range of 300-1000 $\mathrm{nm}$.

Refractive indices of hydrogel samples were measured with optical coherence tomography (central wavelength: $1325 \mathrm{~nm}$; spectral bandwidth: $100 \mathrm{~nm}$ ). Flat samples were produced as described above and placed onto a glass mirror. A B-scan of $3 \times 3 \mathrm{~mm}$ was performed on the edge of the hydrogel, showing both the sample and the reflective mirror surface. The refractive index was calculated as the ratio of optical thickness (distance from upper to lower hydrogel surface) to actual thickness (distance from upper hydrogel surface to mirror surface). Three hydrogels per monomer length and concentration were produced, and three scans were performed per hydrogel.

\subsection{Cell culture}

HEK $293 \mathrm{~T}$ cells were cultured according to a standard protocol in DMEM supplemented with $10 \%$ FCS and $1 \%$ penicillin/streptomycin (all PAN Biotech). To promote cell attachment on PEGDMA hydrogels, a $25 \%$ PEGDMA 8000 gel containing polystyrene particles was produced, and a protein containing layer was added on top of the gel. For this layer, a $50 \%$ PEGDMA 8000 solution was prepared and mixed with the same volume of cold matrigel (Corning), a mixture of extracellular matrix proteins. After protein addition, polymerization was quickly initialized as described above, and the whole solution was added on top of the pre-produced gel as a thin layer. After polymerization, the gel was immersed in cell culture medium and 6000 cells per $\mathrm{mm}^{2}$ were seeded on the modified gel surface. For visualization, cells were stained with green fluorescing Calcein-AM.

\subsection{Optical setup}

Light distribution inside the hydrogels is assessed in a simple optical setup. Light from an interchangeable laser diode is coupled into a biocompatible fiber attached to the hydrogel sample. A sensitive CMOS camera is used for imaging from above (figure 2). Lenses can be interchanged to adjust spot size and image magnification. 
a)

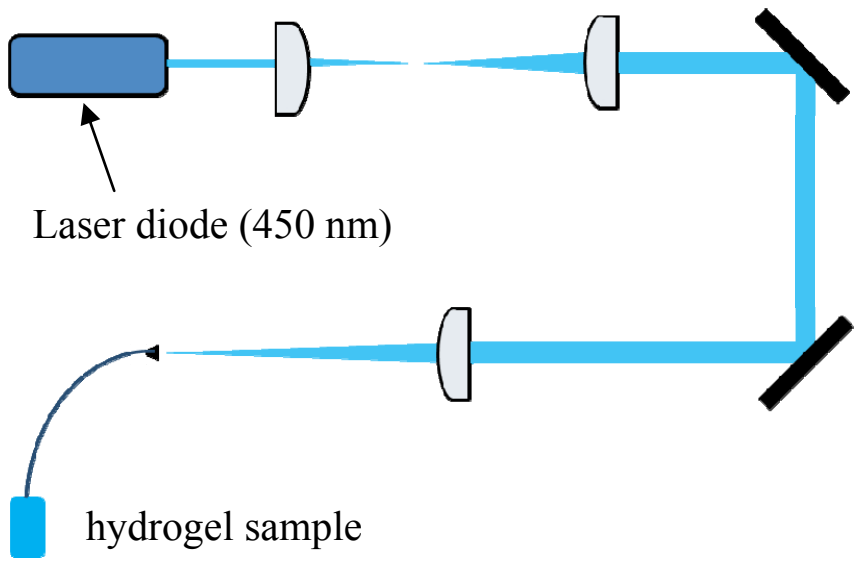

b)

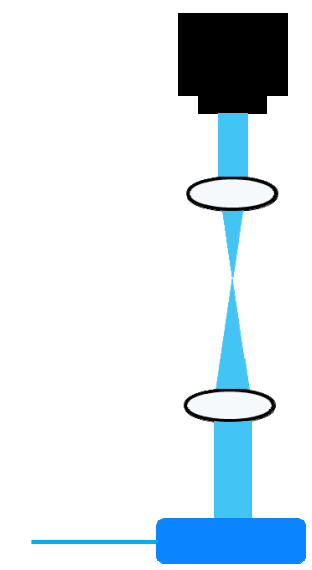

Figure 2: Optical setup to couple light to the hydrogel sample (a) and image light distribution from the top (b). The setup is flexible with regard to the diode's wavelength and power as well as the fiber diameter. A custom sample mount allows for simultaneous cell or tissue culture on the hydrogel.

\section{RESULTS AND DISCUSSION}

\subsection{Overall characteristics of PEGD(M)A hydrogels}

Polymers from poly(ethylene glycol) di(meth)acrylate have tunable properties that vary with monomer length and concentration. The material is biocompatible and bioinert, providing an ideal basis for long term engraftment: the gels are not dissolved or remodeled by the cells' metabolistic activities, and the expected immunomodulation is mild ${ }^{16}$. Long term compatibility of a PEGDA implant on the cardiac muscle has already been demonstrated for regenerative purposes $^{11}$. For the present application it is crucial that the implanted construct retains its overall shape for efficient waveguiding functionality. The PEG derivatives present a good starting point for a biohybrid permanent implant.

Flexibility with simultaneous stability is high for gels from long monomers (MW 8000 and 20,000 in this study), or shorter monomers at low concentrations. With decreasing monomer length and increasing monomer concentration, water content decreases and stiffness of the gels increases. Softness and flexibility are important considerations for successful in vivo engraftment. While quantitative measurements are yet to be done, monomers with a molecular weight of $8000 \mathrm{~g} / \mathrm{mol}$ or more at concentrations of up to $50 \%$ produce gels to generally match several soft tissues ${ }^{8}$.

\subsection{Optical properties}

Loss measurements yielded a damping coefficient of $0.78 \mathrm{~dB} / \mathrm{cm}$ for a gel from PEGDA 700, $90 \%$ at $532 \mathrm{~nm}$. A short monomer at a high concentration is an unfavorable combination for efficient light delivery; with lower concentrations and larger monomers, power loss decreases ${ }^{17}$. However, since the engineered tissue patches are expected to be only a few centimeters in length, a loss of $50 \%$ on a distance of $4 \mathrm{~cm}$ (corresponding to a damping coefficient of $0.78 \mathrm{~dB} / \mathrm{cm}$ ) is already tolerable. A power density of $\sim 1 \mathrm{~mW} / \mathrm{mm}^{2}$ is required to trigger a photocurrent through ChR- $2^{18}$. Current fibercoupled LED devices offer a power output of up to $\sim 20 \mathrm{~mW}$ at the fiber tip ${ }^{19}$. In combination with a low-loss hydrogel waveguide, we expect to reach sufficient radiant exposures to excite cells in direct contact with the hydrogel. Aside from delivering light with high efficiency, functional electrical coupling in the engineered heart muscle tissue is crucial to achieve a wide spread response, strong enough to be passed on to the patient's heart tissue and terminate the fibrillation event. Since the quality of coupling varies, the necessary power output from the hydrogel will have to be determined experimentally.

Transmissivity and refractive index of the hydrogels is primarily dependent on their water content. Long monomers at low concentrations yield gels with a high water content. Transmissivity in most of the visual and the near infrared spectrum is high for these samples (figure 3), while the refractive index approaches the value of water. Tighter polymer 
networks, i.e. from shorter monomers at higher concentrations, possess a higher refractive index. The gels tested during this work yielded values of up to 1.45 . The differences in refractivity are sufficient to achieve total reflection in a gelwithin-gel construct (figure 4).
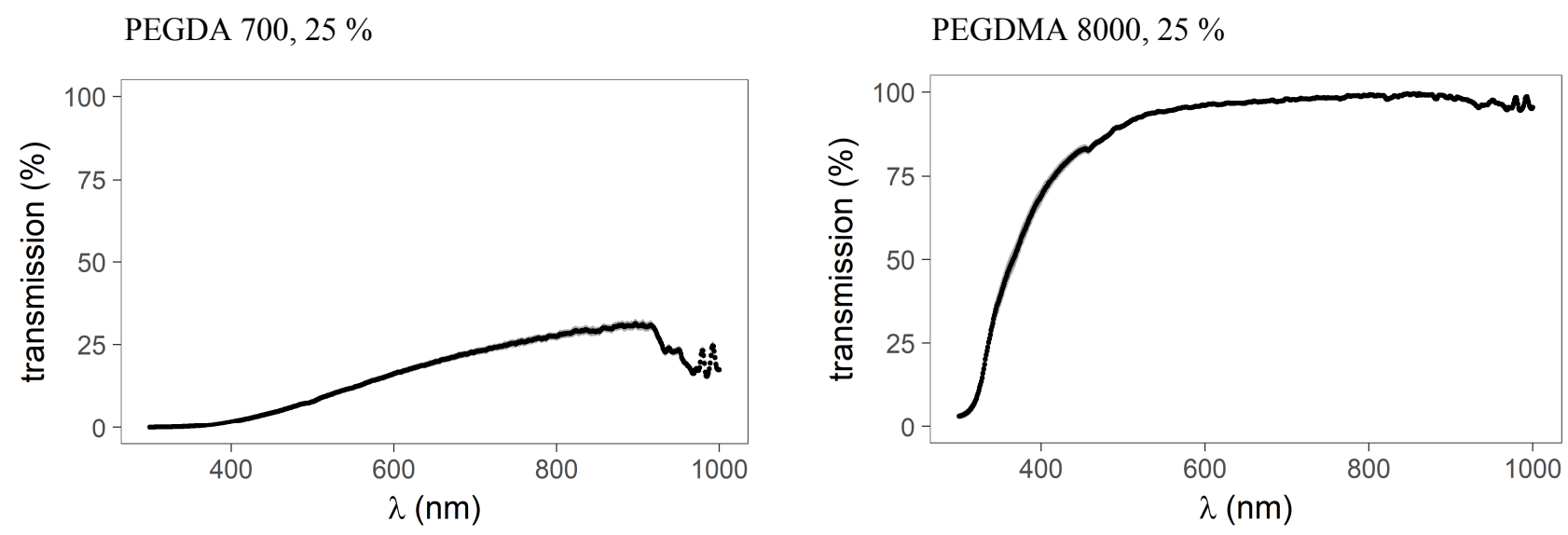

Figure 3: Relative transmission of PEGD(M)A gels in the UV/Vis spectrum. A longer monomer (i.e. a higher water content in the gel) yields gels with considerably higher transmissivity. These gels are favorable for light delivery in optogenetics, were the established variants of ChR-2 are excited at $470 \mathrm{~nm}$ (used in this project) or longer wavelengths of the visible spectrum.

Since the radicalic polymerization reaction which forms the PEGD(M)A hydrogels never consumes all the acrylic head groups, the remaining groups are available to form covalent bonds to a second gel, polymerizing while in contact with the first gel. In this way, a single stable block of material can be manufactured in layers, which can be tuned to differ in their properties such as their refractive index. A fiber-shaped structure inside a gel block can be formed to guide light from a laser or a fiber on a fixed path through the hydrogel (figure 4). Different geometries can be explored to achieve controlled light distribution: bending of the inner hydrogel fiber causes the guided light to leave the fiber structure and propagate into the surrounding gel. Without adding any compounds or further derivatization PEGDA hydrogels allow for controlled light guiding and distribution. However, since modifying a gel's monomer composition also influences its stiffness and transmissivity, the architecture of the whole construct needs to take into account power losses and the hydrogel-tissue interface as well.
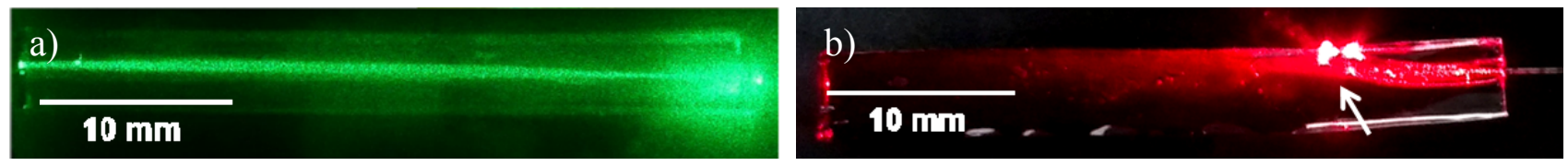

Figure 4: Differences of the gels' refractive indices are large enough to achieve total reflection and controlled waveguiding. a): PEGDA 700, $90 \%$ in a PEGDA 700, $40 \%$ cladding. A laser beam $(532 \mathrm{~nm})$ is coupled into the hydrogel fiber from the right. b): PEGDA 700, $20 \%$ in PEGDMA 20,000, $20 \%$. The short PEGDA 700-fiber is curved, with light leaving this structure at the bend (white arrow).

\subsection{Light distribution}

Waveguiding inside the hydrogels was observed from the top, perpendicular to the direction of fiber attachment (see figure $2 \mathrm{~b}$ ). In the final construct, the light sensitive cells will reside on one flat side of the hydrogel. The light from the fiber should therefore be distributed across the whole area of only one side of the gel. In a simple hydrogel, the light spreads from the inserted fiber on a cone-shaped path and leaves the gel on the opposite side (figure 5a). Cells growing on top such a structure would not be efficiently stimulated. To improve overall light distribution, scattering microparticles were introduced to the hydrogel. Water soluble polystyrene particles were mixed into the PEGDMAsolution prior to polymerization and remained well dispersed after gelation. Light distribution in these gels is more favorable for this application as the scattering particles considerably improve light output from the gel's top side (figure $5 \mathrm{~b}$ ). While optimization of parameters like particle material, size and density is still ongoing, a combination of waveguiding based on changes in refractive index and scattering particles for even light distribution enables us to target a 
large number of cells immobilized on the hydrogel's surface. After optimization of the parameters to achieve the best light distribution in the gel construct, losses have to be reconsidered. Then, the proof of concept will be given by using the scattered light delivered through the hydrogel to evoke a contraction of transgenic heart muscle cells.
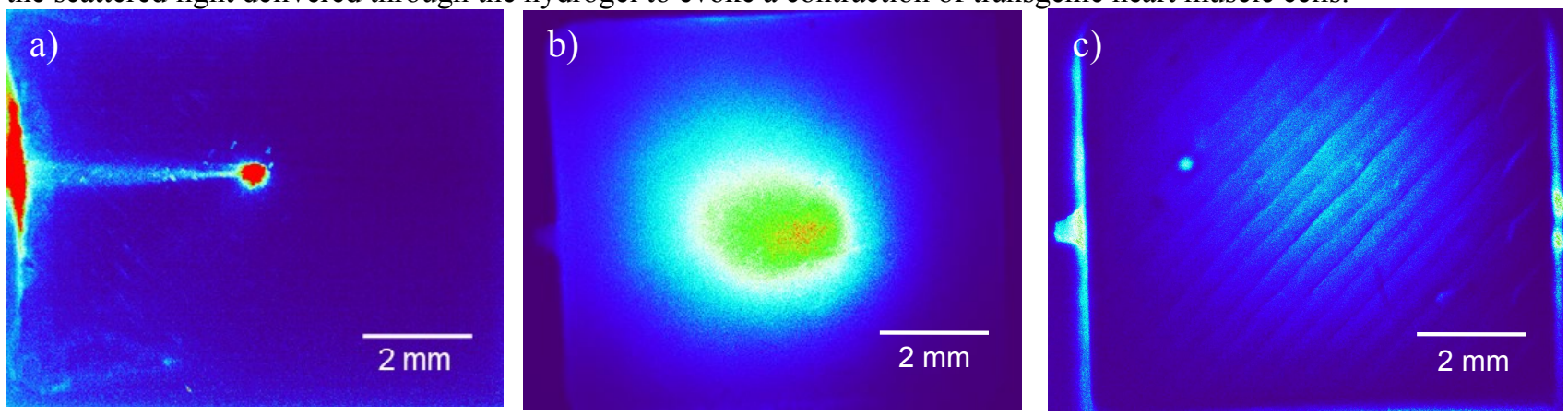

Figure 5: Light distribution (pseudocolor) in PEGDMA gels (MW 8000, $10 \%$ ). a): Brightness was increased to visualize the cone shaped path. The red spot in the center marks the fiber ending. b): Hydrogel containing polystyrene microparticles, top view. Light is distributed in a circle around the fiber ending. c): $\mathrm{TiO}_{2}$ layer of the same hydrogel as b), bottom view. The sheet from titanium oxide particles efficiently reflects the light back into the construct.

In a construct containing polystyrene particles, light is not directed to the upper surface, but scattered in all directions. The final design will be considerably thinner than the gels used for testing, so the lateral surfaces will be narrow. However, losses through the lower surface would be high. We therefore introduced a layer of titanium(IV) oxide $\left(\mathrm{TiO}_{2}\right)$ particles that possess strong backscattering properties and are insoluble in water. This causes the particles to settle to the ground of a forming hydrogel, producing a dense $\mathrm{TiO}_{2}$ layer on the gel's lower surface. This layer effectively scatters the light reaching the bottom of the gel back into the construct, rendering the lower surface considerably darker when observed with the same exposure time as the upper side (figure 5c). For this layer it is especially important that particle distribution remains stable to avoid formation of holes and resulting losses. Long term observations under mechanical stresses mimicking the in vivo environment will be necessary to optimize hydrogel design and performance. One possibility to increase stability of the $\mathrm{TiO}_{2}$ sheet is the addition of another gel on the bottom. Since this surface of the gel will not function as a substrate for cell growth, the mechanics have to be favorable for the construct as a whole, but do not need to match the heart muscle's properties. A stiffer gel, possibly even from a different material, might provide sufficient protection of the construct's functional units.

\subsection{Cell attachment}

PEGD(M)A hydrogels are bioinert and do not offer any features for cells to adhere to. To promote cell attachment at a controlled site, the gels have to be modified. Simple coating of the gels with proteins such as fibrin, collagen or matrigel, a mix of proteins from extracellular matrix, does not provide sufficient stability. Therefore, we exploit the radicalic polymerization reaction to tightly bind proteins to the hydrogel. A thin layer of PEGDMA solution mixed with liquid matrigel is applied on the particle containing gel construct. During polymerization, the PEGDMA molecules bind to the surface of the first gel as well as certain amino groups in the protein mix that are prone to radical formation. In this way, we obtain a PEGDMA layer with immobilized matrix protein, providing anchors for cells to attach to. Cells seeded on top of this protein containing gel can be successfully cultured on the construct, but do not migrate into the deeper layers (figure 6). While long term viability and stability is yet to be demonstrated, this is an easy and versatile method to control hydrogel-tissue interaction. The engineered tissue patch, consisting of matrigel, collagen, fibroblasts and cardiomyocytes, can be immobilized on the hydrogel in the same way. Therefore, the interface can be designed without introducing additional aberrations. 

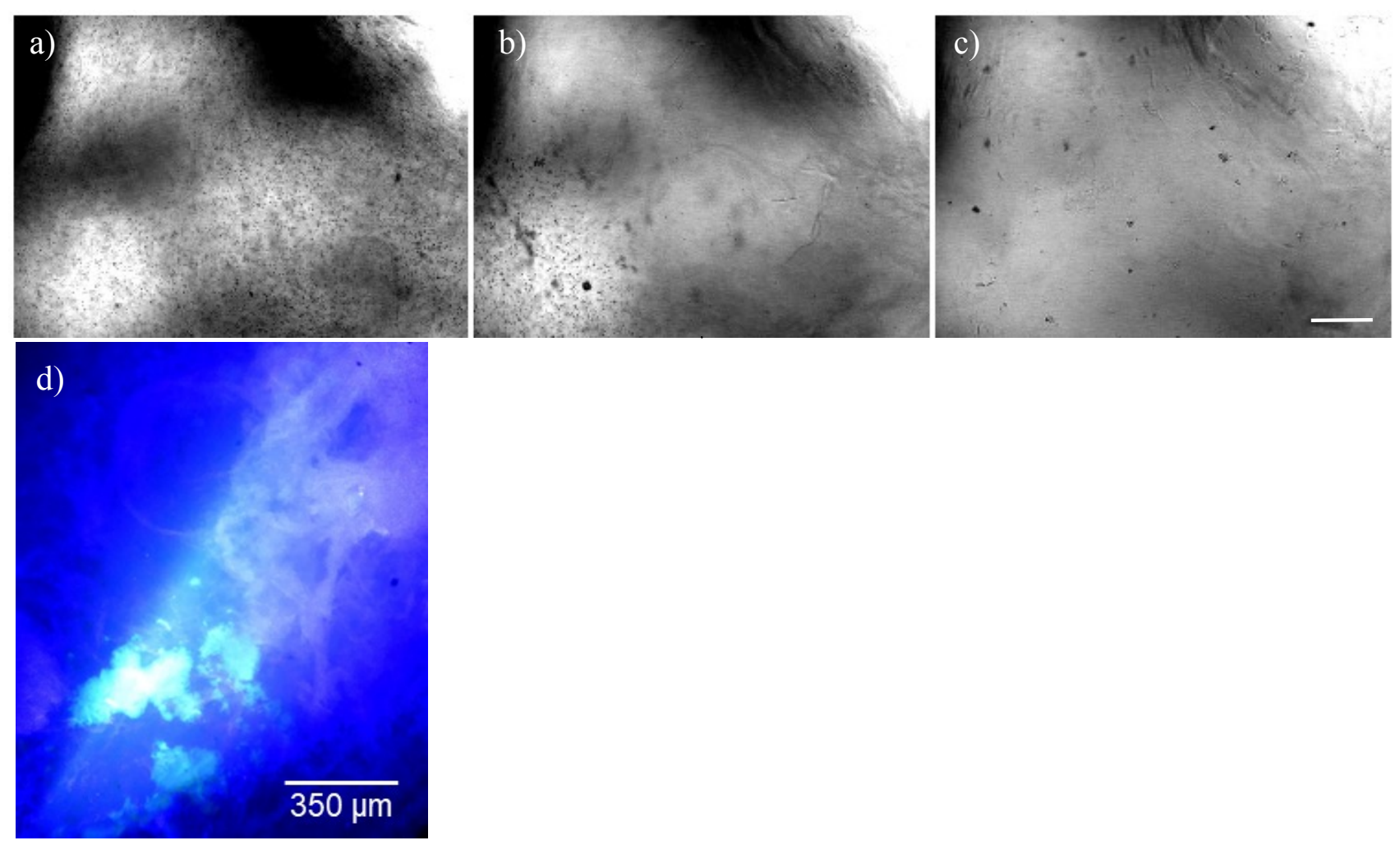

Figure 6: Particle containing hydrogel (MW 8000, 25 \%) with protein layer and HEK cells on top. a-c): Confocal images from different depths, scattering signal. The bottom layer contains polystyrene particles, but no migrating cells were detected. On top of this gel, the protein matrix and attached cells are visible. d): Hydrogel with protein layer and HEK cells, illuminated by the fiber-coupled laser diode. The protein matrix is visible as a foggy structure, cell clusters (stained green) are growing inside the matrix.

\section{CONCLUSION AND OUTLOOK}

We demonstrate a first draft for a hydrogel waveguide for optogenetic applications at the heart muscle. A safe, robust and reliable long term implant will have to match tissue mechanics to ensure stable engraftment and deliver light at sufficient intensity to cells growing on the surface. PEGDMA hydrogels are easily tunable in their mechanical properties by varying monomer length and concentration. However, these parameters also determine the optical properties. A sophisticated layer-by-layer design enables us to achieve controlled waveguiding within the hydrogel while also providing a suitable surface for cell attachment. Introducing scattering microparticles is an easy method to improve light distribution towards the gel surface. Other possibilities to better control light guiding through the construct are internal changes in refractive index, as was demonstrated here as well, or two photon structuring of the hydrogel as has been shown before ${ }^{20,21}$. Laser processing might hold potential for further improvement of our waveguide as well. While optimization and long term validation of the complete construct will take some time, the use of biocompatible, bioinert, modifiable hydrogels for light delivery in vivo is a versatile and promising approach to enable application of optogenetic methods or therapies in living organisms. 


\section{ACKNOWLEDGEMENTS}

This work is funded by the Federal Ministry of Education and Research, Germany, Grant no. 13N14085 and supported by the BIOFABRICATION FOR NIFE Initiative (VWZN2860). Figure 4a was kindly provided by Olga Simon.

\section{REFERENCES}

[1] Deisseroth, K., "Optogenetics," Nat. Methods 8(1), 26-29 (2011).

[2] Govorunova, E. G., Cunha, S. R., Sineshchekov, O. A. and Spudich, J. L., "Anion channelrhodopsins for inhibitory cardiac optogenetics,” Sci. Rep. 6(33530) (2016).

[3] Deisseroth, K., "Optogenetics: 10 years of microbial opsins in neuroscience," Nat. Neurosci. 18, 1213-1225 (2015).

[4] Bruegmann, T., Malan, D., Hesse, M., Beiert, T., Fuegemann, C. J., Fleischmann, B. K. and Sasse, P., "Optogenetic control of heart muscle in vitro and in vivo," Nat. Methods 7(11), 897-900 (2010).

[5] Aravanis, A. M., Wang, L.-P., Zhang, F., Meltzer, L. A., Mogri, M. Z., Schneider, M. B. and Deisseroth, K., "An optical neural interface: in vivo control of rodent motor cortex with integrated fiberoptic and optogenetic technology," J. Neural Eng. 4(3), 143-156 (2007).

[6] Medtronic, ICD Basics, Ask the ICD

http://asktheicd.com/tile/106/english-implantable-cardioverter-defibrillator-icd/how-many-people-have-icds/ (12 February 2018)

[7] Peregrinova, L. and Jordan, J., "Psychotherapy for posttraumatic stress disorders among cardiac patients after implantable cardioverter defibrillator shocks. Feasibility and implementation of a psychocardiological therapy manual in inpatient cardiac rehabilitation," Heart and Mind 1(1), $42-49$ (2017).

[8] Patel, N. R., Whitehead, A. K., Newman, J. J. and Caldorera-Moore, M., "Poly(ethylene glycol) Hydrogels with Tailorable Surface and Mechanical Properties for Tissue Engineering Applications," ACS Biomater. Sci. Eng. 3(8), 1494-1498 (2016)

[9] Choi, M., Woo Choi, J., Kim, S., Nizamoglu, S., Kwang Hahn, S. and Hyun Yun, S., "Light-guiding hydrogels for cell-based sensing and optogenetic synthesis in vivo," Nat. Photonics 7, 987-994 (2013).

[10] Bäckström, S., Benavente, J., Berg, R. W., Stibius, K., Larsen, M. S., Bohr, H. and Helix-Nielsen, C., "Tailoring Properties of Biocompatible PEG-DMA Hydrogels with UV Light," Mater. Sci. Appl.. 3, 425-431 (2012).

[11]Dobner, S., Bezuidenhout, D., Govender, P., Zilla, P. and Davies, N., "A Synthetic Non-degradable Polyethylene Glycol Hydrogel Retards Adverse Post-infarct Left Ventricular Remodeling," J. Card. Fail. 15(7), 629-636 (2009).

[12]Zhu, J. and Marchant, R. E., "Design properties of hydrogel tissue-engineering scaffolds," Expert Rev. Med. Devices 8(5), 607-626 (2014).

[13]Zhang, X., Xia, L. Y., Chen, X., Chen, Z. and Wu, F.-G., "Hydrogel-based phototherapy for fighting cancer and bacterial infection," Sci. China Mater. 60(6), 487-503 (2017).

[14]Kensah, G., Lara, A. R., Dahlmann, J., Zweigerdt, R., Schwanke, K., Hegermann, J., Skvorc, D., Gawol, A., Azizian, A., Wagner, S., Maier, L. S., Krause, A., Dräger, G., Ochs, M., Haverich, A., Gruh, I. and Martin, U., "Murine and human pluripotent stem cell-derived cardiac bodies form contractile myocardial tissue in vitro," Eur. Heart J. 34, 1134-1146 (2013).

[15] Bruegmann, T., Boyle, P. M., Vogt, C. C., Karathanos, T. V., Arevalo, H. J., Fleischmann, B. K., Trayanova, N. A. and Sasse, P., "Optogenetic defibrillation terminates ventricular arrhythmia in mouse hearts and human simulations," J. Clin. Invest. 126(10), 3894-3904 (2016).

[16] Drury, J. L. and Monney, D. J., "Hydrogels for tissue engineering: scaffold design variables and applications," Biomaterials 24, 4337-4351 (2003).

[17] Choi, M., Choi, J. W., Kim, S., Nizamoglu, S., Hahn, S. K. and Yun, S. H., "Light-guiding hydrogels for cellbased sensing and optogenetic synthesis in vivo," Nat. Photonics 7, 987-994 (2013).

[18] Mattis, J., Tye, K. M., Ferenczi, E. A., Ramakrishnan, C., O’Shea, D. J., Prakash, R., Gunaydin, L. A., Hyun, M., Fenno, L. E., Gradinaru, V., Yizhar, O. and Deisseroth, K., "Principles for applying optogenetic tools derived from direct comparative analysis of microbial opsins," Nat. Methods 9(2), 159-172 (2012). 
[19] Thorlabs, Fiber-coupled LEDs

https://www.thorlabs.com/newgrouppage9.cfm?objectgroup id=5206 (12 February 2018)

[20] Ciuciu, A. I. and Cywiński, P. J., "Two-photon polymerization of hydrogels - versatile solutions to fabricate well-defined 3D structures," RSC Adv. 4, 45504-45516 (2014).

[21] Lee, S.-H., Moon, J. J. and West, J. L., "Three-dimensional micropatterning of bioactive hydrogels via twophoton laser scanning photolithography for guided 3D cell migration," Biomaterials 20, 2962-2968 (2008). 